\section{Significance of gene expression analysis in uveal melanoma in comparison to standard risk factors for risk assessment of subsequent metastases}

U Petrausch ${ }^{1,7}$, P Martus $^{2}, \mathrm{H}_{\text {Tönnies }}^{3}$, NE Bechrakis ${ }^{4}$, D Lenze ${ }^{5}$, S Wansel ${ }^{4}$, M Hummel ${ }^{5}$, N Bornfeld ${ }^{6}$, E Thiel $^{1}$, MH Foerster ${ }^{4}$ and U Keilholz ${ }^{1}$
'Department of

Medicine III (Hematology, Oncology and Transfusion Medicine), Charité, Campus Benjamin Franklin,

Germany

${ }^{2}$ Department of Biometry and Clinical Epidemiology, Charité, Campus

Benjamin Franklin, Germany

assess for the risk of subsequent metastases. Eye (2008) 22, 997-1007; doi:10.1038/sj.eye.6702779; published online 23 March 2007

Keywords: uveal melanoma; gene expression profile; comparative genomic hybridisation; risk factors

\section{Introduction}

Uveal melanoma can be locally treated using a variety of methods, but there is no indication that any of these modalities will influence the risk for development of hematogenous spread, usually to the liver, ${ }^{1}$ which in almost all patients will be fatal within months ${ }^{2,3}$ to years. ${ }^{4}$ Thus, there are two basic groups of patients according to clinical course, namely patients in whom the tumor will remain confined to the eye, and a second group of patients who will develop hematogenous metastases during follow up. Owing to the absence of lymphatic drainage of the uvea, there is no relevant third group of patients with lymphatic spread. This two-group model should allow comparison of different risk factors from different data sources in relatively small patient cohorts.

A number of clinical and histological risk factors have been defined over the last 3 decades, among these are the clinico-pathological factors like location, extraocular growth, ciliary body (CB) involvement and the epitheloid-cell tumor type..$^{5-9}$ Presence of tyrosinase transcripts in
${ }^{3}$ Department of Human Genetics, Charité,

Campus Virchow-Kliniken, Berlin, Germany

${ }^{4}$ Department of Ophthalmology, Charité, Campus Benjamin Franklin, Berlin, Germany

${ }^{5}$ Department of Pathology, Charité, Campus Benjamin Franklin, Berlin, Germany

${ }^{6}$ Division of Ophthalmology, Essen, Germany

Correspondence: U Keilholz, Tel: + 493084453906 ; Fax: + 493084454021 . E-mail: ulrich.keilholz@ charite.de

${ }^{7}$ Current address: Laboratory of Molecular and Tumor Immunology, Robert Franz Cancer Center, Earle A Chiles Research Institute, Providence Cancer Center and Providence Portland OR 97213, USA

Received: 8 July 2006 13 February 2007 Published online: 23 March 2007 University Hospital Essen, Medical Center, Portland, Accepted in revised form: 
peripheral blood, suggesting circulating melanoma cells, has also been linked to subsequent appearance of metastases. ${ }^{10,11}$ Almost 10 years ago, monosomy 3 was defined as a strong chromosomal risk factor, ${ }^{12}$ which appeared to be superior to all clinical and histological factors in the prediction of subsequent metastasis.

Subsequently, gene expression profiling has defined gene expression patterns that are associated with monosomy $3^{13}$ and recently, gene expression patterns, which are associated with the development of metastases independent of clinical risk factors. ${ }^{14}$

We here report on a comprehensive approach comparing gene expression profiling and comparative genomic hybridization (CGH) with clinical and histological data for all analyzed patients. The analysis was performed in a group of 28 patients with primary uveal melanoma with a follow-up ranging from 3 to 7 years, who had received enucleation or tumor excision as primary treatment for large uveal melanoma. The comprehensive approach with discriminant analysis of the same type for clinico-pathological, chromosomal and gene expression data offers the possibility to compare the value of each data set for the classification of patients into groups according to their risk for the development of subsequent metastases. The clinico-pathological data was separately analyzed for the prediction of metastases-free survival. In addition, the value for risk assessment of subsequent metastasis of published gene expression data associated with monosomy $3^{13}$ and predicting metastatic phenotype ${ }^{14}$ respectively, was verified.

\section{Materials and methods}

\section{Patients and tumor samples}

Patients with uveal melanoma from our hospital were included into this study, if shock-frozen tissue was available from enucleation or tumor excision as primary therapy, and if the follow-up period since primary surgery exceeded 3 years. The investigation was approved by the institutional ethic committee. The clinical characteristics of the primary uveal melanoma considered included tumor localization, CB involvement, extraocular tumor growth, largest tumor diameter, tumor height, and dominant cell type. The patient's charts were reviewed and the patient's ophthalmologist and/or general physicians were contacted to verify follow-up information.

\section{Comparative genomic hybridization}

DNA was extracted out of banked primary tumor tissue using the Genomic DNA Purification Kit (Gentra, Minneapolis, MN, USA) according to the manufacturer's instructions. CGH technique was performed as described previously ${ }^{15}$ with slight modifications. Extracted DNA was labeled by nick-translation using direct SpectrumGreen ${ }^{\circledR}$ (Test-DNA) and SpectrumOrange ${ }^{\circledR}$ (Reference DNA) -conjugated dUTP (VYSIS, Downers Grove, IL). Because of low DNA amount, five samples (150-99; 274-97; 193-97; 226-97 and 047-00) had to be amplified using a whole genome PCR amplification (Expand High Fidelity PCR System ${ }^{\mathbb{R}}$; HIFI-DOP; Roche, Penzberg, Germany; for protocol details see manufacturer's instructions). Technical controls showed no differences to the standard protocol. Amplified test-DNAs were also labeled by nick translation.

Target metaphase spreads were prepared from PHA-stimulated peripheral blood lymphocytes from a karyotypically normal male. Slides were dehydrated in 70,80 and $95 \%$ ethanol, denatured for $2 \mathrm{~min}$ in $70 \%$ formamide $/ 2 \times \mathrm{SSC}, \mathrm{pH} 7$, at $70-72^{\circ} \mathrm{C}$, followed by further dehydration in ice cold ethanol. For each hybridization, $200 \mathrm{ng}$ of labeled test DNA, $200 \mathrm{ng}$ reference DNA, and $12.5 \mu \mathrm{g}$ Cot-1 DNA were mixed and ethanol precipitated. DNA was resuspended in $14 \mu \mathrm{l}$ hybridization mix containing 50\% formamide, $2 \times$ SSC and $10 \%$ dextran sulphate, denatured at $70^{\circ} \mathrm{C}$ for $5 \mathrm{~min}$ and applied to denatured metaphase spreads. Slides were incubated at $37^{\circ} \mathrm{C}$ in a moist chamber for 2 days. Standard posthybridization washes were performed. The slides were counterstained with 4', 6-diamino-2-phenylindole dihydrochloride (DAPI) and mounted with antifading solution (Vectashield, Vector Laboratories, Inc. Burlingame, CA, USA). For each CGH-analysis, 10 metaphases have been captured. Ratio profiles have been calculated after karyotyping of six non-overlapping metaphase spreads (12 single chromosomes for each homologue).

\section{Digital image analysis}

CGH hybridizations were analyzed using an epifluorescence microscope (Axioscope, Zeiss, Germany) fitted with different single band pass filter sets for DAPI (blue), Spectrum Green ${ }^{\mathbb{R}}$ (green) and Spectrum Orange ${ }^{\mathbb{R}}$ (red) fluorescence. The microscope was equipped with an integrated high-sensitivity monochrome chargecoupled device camera (Hamamatsu, Shizuoka, Japan) for image acquisition. Image analysis and karyotyping was performed with ISIS digital image analysis system (Metasystems, Altlussheim, Germany). Captured metaphases were karyotyped and analyzed to detect chromosomal imbalances on each chromosome. Diagnostic thresholds of 0.85 and 1.17 were used for the identification of chromosomal under-representations (losses) and over-representations (gains). 


\section{RNA preparation and affymetrix genechip hybridization}

Total RNA was extracted from banked primary tumor tissue using the RNeasy kit (Qiagen, Hilden, Germany) according to the manufacturer's instructions. Total RNA was screened for RNA degradation with the Agilent BioAnalyzer (Agilent, Palo Alto, CA, USA). Only samples with no or weakest signs of RNA degradation were used. Samples were analyzed on high-density microarrays (HG-U133A, Affymetrix, Santa Clara, CA, USA). Because of a relatively small total RNA yield in some samples, all samples were subjected to Affymetrix 'small sample protocol' version II target preparation (Affymetrix). The RNA amplification procedure is known to be associated with shorter cRNA fragments as compared to the standard protocol. A lower length of cRNA fragments can be demonstrated by higher $3^{\prime} / 5^{\prime}$ ratios for GAPDH and beta-actin documented in the Affymetrix report file. The median $3^{\prime} / 5^{\prime}$ ratio for all 28 samples of GAPDH was 4.6 (2-10.9), and the median for $\beta$-actin was 14.4 (2.2-76) suggesting suboptimal amplification towards the $5^{\prime}$ end in a proportion of samples. Further, the $3^{\prime} /$ midratios of GAPDH and $\beta$-actin were analyzed, which are less influenced by the amplification protocol and more relevant for the $3^{\prime}$-based analysis. The median $3^{\prime} /$ midratio of all samples for GAPDH was 2 (1.2-3.2), and the median for $\beta$-actin was 3.9 (0.8-10.9), indicating that the bias was largely confined towards the $5^{\prime}$ end, and that the sample quality for the $3^{\prime}$ region based gene expression profiling was adequate for all samples. All signal values, all p-detection values and technical control data are online according to MIAME standard [Internet address: http:// www.charite.de/haema-cbf/labor/AGKeilholz.htm].

\section{Data analysis}

Time to metastases was analysed using the Kaplan-Meier method and log rank tests. Gene expression data was preprocessed by MAS 5.0 (Affymetrix). Signal values and p-detection values of 22283 probe sets were available. Sixty-eight probe sets for technical controls were excluded. For the following calculations signal values and p-detection values of 22215 probe sets were used. Signal values with $\mathrm{p}$-detection value (against background) $>0.02$ were defined as irrelevant. Probe sets entered the statistical analysis if at least 13 samples per probe set were classified as relevant. This classification was performed without use of information about the status of subjects (with or without metastases).

Chromosomal data were coded as +1 (intensity $>1.17$ ), 0 (intensity between 0.85 and 1.17 ) and -1 (intensity <0.85). Gender was coded as $1 / 2$ (female/ male), metastases, ciliary body involvement (CB), and extraocular tumor growth EOE as 0/1 (no/yes), tumor type $1 / 2 / 3$ (epitheloid, mixed, spindle), largest tumor diameter (LTD), tumor height, and age at diagnosis were coded as quantitative variables.

The results include:

(1) Descriptive analyses. Patient cohorts, clinical and pathological data, and chromosomal events.

(2) Primary analysis (comparative supervised learning). The primary aim of the statistical analysis was the comparison between the three subsets of potential predictors (gene expression data, chromosome data, clinicopathological data) with regard to their prognostic value. To this aim, the robust method of diagonal linear discriminant analysis ${ }^{16}$ with crossvalidation was applied to each of the three subsets separately. To avoid bias, crossvalidated classifiers were constructed using the leaving one out'-method. ${ }^{16}$ Classifiers were standardized so that positive values corresponded to a correct classification for patients, both with and without metastases. The size of the values further quantified the confidence of this classification. Finally, the classifiers were compared between the three sets of prognostic variables using the Wilcoxon test for paired samples. One set of prognostic variables was considered superior to another one if the values of this classifier were significantly larger than those of the other one.

Additionally, the following secondary analyses were performed:

(3) Unsupervised learning for all probe sets: Cluster analysis with squared euclidean distance and average linkage aggregation was applied for all probesets not classified as irrelevant according to their p-detection value against background.

(4) Classifying genes: Additionally, based on preselected probe sets in each crossvalidation step of the gene expression data the set $S_{i}$ was determined, which contained 100 probes with the highest absolute standardized difference among all 8465 preselected probe sets. Fifty probes were present in all 28 sets $S_{i}$ and titled as classifying genes. For descriptive purpose only, a second cluster analysis was performed with the 50 classifying probe sets. (Genespring software: hierarchical cluster analysis, standard correlation). As it was not the primary aim of our analysis to identify classifying genes, genes were not selected according to a prespecified value of the Force Detection Rate (FDR).

(5) Supervised learning using published gene lists: The published gene list associated with monosomy 3 were transferred from the Affymetrix GeneChip HG-U95v2 coding (201 probe sets [Internet address: 
http://www.uni-essen.de/humangenetik/

download]) to the Affymetrix GeneChip HG-U133A coding (175 probe sets) using Affymterix algorithm 'best match' (NetAffx [Internet address: http:// www.affymetrix.com/index.affx]). The gene list associated with metastatic phenotype (62 genes) was transformed into $101 \mathrm{HG}-\mathrm{U} 133 \mathrm{~A}$ probe sets. Both probe set lists were used as classifiers for discriminant analysis. No preselection regarding p-detection value (against background) was performed and the construction of classifiers was performed using cross-classification as described for the primary analysis.

All analyses were performed using R, SPSSWIN (release 11.5) and Genespring 4.2 (Silicon Genetics, Redwood City, CA, USA). Probe sets were labeled with gene titles by using Affymetrix website NetAffx [Internet address: http://www.affymetrix.com/index.affx].

\section{Results}

\section{Patient cohort}

Out of 32 patients identified to fulfill entry criteria for the analysis, 28 patients could be included into the study.

Reasons for non-inclusion were insufficient DNA $(n=2)$ or RNA $(n=2)$ quality. All 28 patients had received enucleation or tumor excision as primary treatment and 12 patients had developed subsequent metastases. The median follow-up since primary treatment was 68 months, ranging from 37 months to 91 months. The patient characteristics are shown in Table 1.

\section{Clinical and pathological data}

The entire patient cohort had large tumors requiring enucleation or excision. The clinico-pathological data listed in Table 1 were analyzed by univariate KaplanMeier survival analysis. Metastases-free survival was

Table 1 Patients characteristics

\begin{tabular}{|c|c|c|c|c|c|c|c|c|c|}
\hline No. & Sex & Metastases & Type & $\begin{array}{l}L T D \\
(\mathrm{~mm})\end{array}$ & $\begin{array}{l}\text { Tumor height } \\
\text { (mm) }\end{array}$ & $E O E$ & $C B$ & $\begin{array}{l}\text { Follow-up } \\
\text { (months) }\end{array}$ & $\begin{array}{c}\text { Age at treatment } \\
\text { (years) }\end{array}$ \\
\hline 005-97 & $\mathrm{F}$ & Yes & Mixed & 18 & 7 & No & Yes & 83 & 31 \\
\hline 130-96 & F & Yes & Epitheloid & 11 & 9.5 & Yes & No & 83 & 55 \\
\hline 098-99 & $\mathrm{M}$ & Yes & Mixed & 15 & 13 & Yes & No & 47 & 55 \\
\hline 075-99 & M & Yes & Mixed & 10 & 12 & No & No & 49 & 54 \\
\hline 154-99 & $\mathrm{M}$ & Yes & Epitheloid & 22 & 15 & No & Yes & 43 & 61 \\
\hline 178-97 & $\mathrm{F}$ & Yes & Epitheloid & 17 & 10 & No & No & 77 & 62 \\
\hline 071-01 & M & Yes & Spindle & 16 & 8 & No & Yes & 37 & 55 \\
\hline 193-97 & M & Yes & Spindle & 17 & 9 & No & No & 71 & 71 \\
\hline 226-97 & $\mathrm{F}$ & Yes & Mixed & 16 & 9 & No & No & 69 & 61 \\
\hline 147-99 & $\mathrm{F}$ & Yes & Mixed & 21 & 13 & No & Yes & 43 & 62 \\
\hline 127-99 & M & Yes & Mixed & 18 & 9 & No & Yes & 39 & 47 \\
\hline $047-00$ & $\mathrm{~F}$ & Yes & Mixed & 17 & 9 & No & No & 45 & 61 \\
\hline 274-97 & F & No & Spindle & 9 & 6 & No & No & 69 & 74 \\
\hline 139-97 & M & No & Spindle & 11 & 9 & No & No & 73 & 45 \\
\hline $174-96$ & M & No & Mixed & 23 & 11 & No & No & 86 & 67 \\
\hline 013-99 & M & No & Spindle & 12 & 4 & No & No & 58 & 35 \\
\hline 097-98 & M & No & Mixed & 21 & 12 & No & Yes & 61 & 59 \\
\hline $187-96$ & M & No & Spindle & 17 & 9.5 & No & No & 83 & 55 \\
\hline 026-97 & M & No & Spindle & 21 & 14 & No & Yes & 78 & 54 \\
\hline 179-96 & $\mathrm{F}$ & No & Spindle & 20 & 12 & No & No & 88 & 54 \\
\hline $108-97$ & M & No & Epitheloid & 19 & 17 & No & No & 80 & 66 \\
\hline 051-98 & M & No & Mixed & 16 & 7 & No & Yes & 68 & 47 \\
\hline 001-98 & F & No & Spindle & 13 & 9 & No & Yes & 68 & 57 \\
\hline 219-96 & M & No & Spindle & 12 & 11 & No & No & 91 & 74 \\
\hline 150-99 & M & No & Mixed & 11 & 7 & No & No & 46 & 52 \\
\hline $135-98$ & M & No & Mixed & 19 & 12 & No & Yes & 51 & 62 \\
\hline $101-99$ & M & No & Mixed & 15 & 9 & No & No & 53 & 37 \\
\hline 210-97 & M & No & Mixed & 11 & 7.5 & No & Yes & 70 & 41 \\
\hline \multirow[t]{2}{*}{ Total } & M: 19 & Yes: 12 & Spindle: 10 & & & Yes: 2 & Yes: 11 & & \\
\hline & F: 9 & No: 16 & $\begin{array}{l}\text { Epitheloid: } 4 \\
\text { Mixed: } 14\end{array}$ & & & No: 26 & No: 17 & & \\
\hline Max & & & & 23 & 17 & & & 91 & 74 \\
\hline Median & & & & 16.5 & 9.25 & & & 68 & 55 \\
\hline Min & & & & 9 & 4 & & & 37 & 31 \\
\hline
\end{tabular}

$\mathrm{CB}$, ciliary body involvement; EOE, extraocular growth; LTD, largest tumor diameter.

Female (F), male (M); patients with (yes) and without (no) subsequently developed metastases; dominant cell type (type). 
significantly shorter in female as compared to male patients $(P<0.02)$ and in patients with EOE as compared to patients without $\mathrm{EOE}(P<0.002)$, but EOE was diagnosed only in two patients. No significant difference in metastases-free survival was detected analyzing $\mathrm{CB}$ involvement $(P<0.85)$, largest tumor diameter $(P<0.54)$, tumor height $(P<0.73)$, age at treatment $(P<0.85)$ and different dominant cell types $(P<0.13)$.

\section{Chromosomal events}

Chromosomal gains and losses are summarized in Figure 1. The most frequent events involved chromosomes 3, 6, 8, and Y. A complete loss of one chromosome 3 was found in 11 of 28 cases including nine of 12 cases with subsequent metastases, supporting the major role of monosomy 3 for metastatic phenotype. Interestingly, one sample (047-00) from a patient with metastases revealed trisomy 3 . Two samples from patients with metastases (226-97, 193-97) displayed no aberration of chromosome 3 . The gene expression pattern of samples $047-00$ and 226-97 were grouped by discriminant analysis (see below) to patients with metastases whereas sample 193-97 was classified to patients without metastases. In the samples from two patients without subsequent metastases (210-97 and 108-97) monosomy 3 was detectable. The first of these samples was classified to the group with subsequent metastases, whereas the second was classified to the group without subsequent metastases.

Variable partial chromosomal gains in the short arm of chromosome 6 and one complete trisomy of chromosome 6 were exclusively detected in the group of patients without subsequent metastases (nine of 16 patients without subsequent metastases). Among these was one patient (108-97) with a 6p gain coinciding with monosomy 3 and no metastases. Complete trisomy 8 was detected in three patients without metastases, including two patients with monosomy 3 without metastasis, whereas loss of the short arm of chromosome 8 occurred in five patients with monosomy 3 and metastatic disease. This finding indicates in our study that chromosomal gains in chromosome $6 \mathrm{p}$ and $8 \mathrm{p}$ are associated with a better outcome of the patients.

\section{Data analysis}

Preselection of genes

On the basis of the distribution of p-detection values a total of 8465 probe sets were preselected with a p-detection value (against background) of below 0.02 in at least 13 samples.
Primary analysis (supervised learning): comparison of gene expression data and chromosomal events to clinico-pathological data as prognostic factors

Comparison of the grouping precision by diagonal linear discriminant analysis with crossvalidation yielded a significant ranking between gene expression, chromosomal, and clinicopathological data in classifying patients corresponding to development of metastases. Discriminant analysis applied to gene expression data grouped 26 of 28 patients correctly (Figure 2c). The same type of analysis applied to CGH data grouped 23 of 28 samples correctly, and when applied to the clinico-pathological data, 16 patients were grouped correctly (Figure $2 \mathrm{a}$ and $\mathrm{b}$ ). Considering the quantitative discriminant scores, the grouping precision of the gene expression analysis was significantly superior to chromosomal data $(P<0.01)$ and to clinico-pathological factors $(P<0.001$, Figure $2 d)$.

\section{Secondary analysis (unsupervised learning)}

The unsupervised analysis based on 8465 probe sets yielded a classification into two groups (groups A and B), with 12 of 11 patients with subsequent development of metastases clustering in group A, and 14 of 15 patients without metastases clustering in group B. Sample 210-97, which is one of the two samples without subsequent development of metastases and monsomy 3, was separately grouped (Figure 3).

\section{Secondary analysis (gene selection)}

To identify the most prominent classifying genes, that is the genes differentially expressed in all samples of metastasizing vs non-metastasizing tumors, the 100 probe sets with the highest absolute standardized difference among all 8465 preselected probe sets were considered. Of these, 50 probe sets were detected as classifiers on the basis that they appeared in every step of the 'leaving one out'-method crossvalidation analysis. These defined 50 probe sets were annotated and 42 genes were identified. To visualize the gene expression pattern of the 50 classifying probe sets a hierarchical cluster analysis was performed and yielded a classification into two groups (Figure 4).

\section{Secondary analysis (application of published gene lists as classifier)}

To analyze the classification accuracy of the published gene lists by Tschtenscher ${ }^{13}$ and Onken ${ }^{14}$ in our own data set, gene lists were transformed to coding of HG-133A Affymetrix GeneChip and further used as classifier for diagonal discriminant analysis. On the level of probe set identifiers an overlap of five discriminant genes was found compared to a gene list associated with monosomy $3^{13}$ and an overlap of four discriminant genes compared 


\begin{tabular}{|c|c|c|c|c|c|c|c|c|c|c|c|c|c|c|c|c|c|c|c|c|c|c|c|c|}
\hline metastases & $n$ & $n$ & \begin{tabular}{l|l}
$n$ & 1
\end{tabular} & $n$ n & $n$ & $\mathrm{n}$ & $n$ & \begin{tabular}{l|l}
$n$ & $n$
\end{tabular} & $n$ & $\mathrm{n}$ & $\mathrm{n}$ & $n$ & \begin{tabular}{l|l}
$n$ & $r$
\end{tabular} & $\mathrm{n} \mid \mathrm{y}$ & $\mathrm{y}$ & $\mathrm{y}$ & $y$ & \begin{tabular}{l|l}
$y$ & $y$
\end{tabular} & $y$ & $y$ & $y$ & \begin{tabular}{l|l}
$\mathrm{y}$ & $\mathrm{y}$
\end{tabular} & $y$ & \\
\hline & \begin{tabular}{l}
$\hat{\alpha}$ \\
$\dot{\alpha}$ \\
0 \\
0 \\
\hdashline
\end{tabular} & 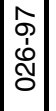 & \begin{tabular}{l|l}
$\hat{s}$ & \\
oे \\
N
\end{tabular} & 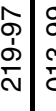 & $\hat{s}$ & 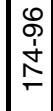 & \begin{tabular}{|c|} 
\\
$\$$ \\
1 \\
$\infty$ \\
- \\
\end{tabular} & 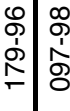 & 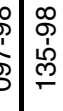 & \begin{tabular}{|c|} 
\\
\\
0 \\
0 \\
0 \\
-1 \\
\end{tabular} & $\begin{array}{l}\text { o } \\
\text { o } \\
\frac{1}{8} \\
8\end{array}$ & $\begin{array}{l}0 \\
o \\
\frac{1}{20} \\
\frac{1}{0}\end{array}$ & 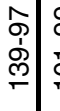 & & \begin{tabular}{|l|l}
8 \\
$\stackrel{1}{5}$ \\
5 \\
\end{tabular} & 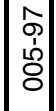 & 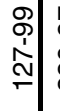 & & 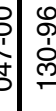 & 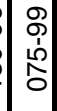 & $\begin{array}{l}8 \\
1 \\
1 \\
0\end{array}$ & & 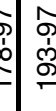 & \\
\hline Chromosome & & & & & & & & & & & & & & & & & & & & & & & & \\
\hline $1 p$ & & & & & & & & & & & & & & & & & & & & & & & & $1 p$ \\
\hline $1 q$ & & & & & & & & & & & & & & & & & & & & & & & & $1 q$ \\
\hline $2 p$ & & & & & & & & & & & & & & & & & & & & & & & & $2 p$ \\
\hline $2 q$ & & & & & & & & & & & & & & & & & & & & & & & & $2 q$ \\
\hline $3 p$ & & & & & & & & & & & & & & & & & - & & & & & & & $3 p$ \\
\hline $3 q$ & & & & & & & & & & & & & & & & & & & & & & & & $3 q$ \\
\hline $4 p$ & & & & & & & & & & & & & & & & & & & & & & & & $4 p$ \\
\hline $4 q$ & & & & & & & & & & & & & & & & & & & & & & & & $4 q$ \\
\hline $5 p$ & & & & & & & & & & & & & & & & & & & & & & & & $5 p$ \\
\hline $5 q$ & & & & & & & & & & & & & & & & & & & & & & & & $5 q$ \\
\hline $6 p$ & & & & & & & & & & & & & & & & & & & & & & & & $6 p$ \\
\hline $6 q$ & & & & & & & & & & & & & & & & & & & & & & & & $6 q$ \\
\hline $7 p$ & & & & & & & & & & & & & & & & & & & & & & & & $7 p$ \\
\hline $7 q$ & & & & & & & & & & & & & & & & & & & & & & & & $7 q$ \\
\hline $8 p$ & & & & & & & & & & & & & & & & 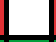 & & & & & & & - & $8 p$ \\
\hline $8 q$ & & & & & & & & & & & & & & & & 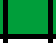 & - & & & & & & - & $8 q$ \\
\hline $9 p$ & & & & & & & & & & & & & & & & & - & & & & & & 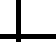 & $9 p$ \\
\hline $9 q$ & & & & & & & & & & & & & & & & & 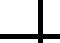 & & & & & & & $9 q$ \\
\hline $10 p$ & & & & & & & & & & & & & & & & & 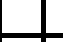 & & & & & & & $10 p$ \\
\hline $10 q$ & & & & & & & & & & & & & & & & & 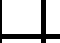 & & & & & & & $10 \mathrm{q}$ \\
\hline $11 p$ & & & & & & & & & & & & & & & & & 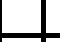 & & & & & & & $11 p$ \\
\hline $11 q$ & & & & & & & & & & & & & & & & & 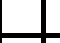 & & & & & & & $11 \mathrm{q}$ \\
\hline $12 p$ & & & & & & & & & & & & & & & & & 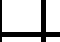 & & & & & & & $12 p$ \\
\hline $12 q$ & & & & & & & & & & & & & & & & & & & & & & & & $12 q$ \\
\hline $13 q$ & & & & & & & & & & & & & & & & & & & & & & & & $13 q$ \\
\hline $14 q$ & & & & & & & & & & & & & & & & & & & & & & & & $14 q$ \\
\hline $15 q$ & & & & & & & & & & & & & & & & & & & & & & & & $15 q$ \\
\hline $16 p$ & & & & & & & & & & & & & & & & & & & & & & & & $16 p$ \\
\hline $16 q$ & & & & & & & & & & & & & & & & & & & & & & & & $16 \mathrm{q}$ \\
\hline $17 p$ & & & & & & & & & & & & & & & & & & & & & & & & $17 p$ \\
\hline $17 q$ & & & & & & & & & & & & & & & & & & & & & & & & $17 q$ \\
\hline $18 p$ & & & & & & & & & & & & & & & & & & & & & & & & $18 p$ \\
\hline $18 \mathrm{q}$ & & & & & & & & & & & & & & & & & & & & & & & & $18 q$ \\
\hline $19 p$ & & & & & & & & & & & & & & & & & & & & & & & & $19 p$ \\
\hline $19 q$ & & & & & & & & & & & & & & & & & & & & & & & & $19 q$ \\
\hline $20 p$ & & & & & & & & & & & & & & & & & & & & & & & & $20 p$ \\
\hline $20 q$ & & & & & & & & & & & & & & & & & & & & & & & & $20 q$ \\
\hline $21 q$ & & & & & & & & & & & & & & & & & & & & & & & & $21 \mathrm{q}$ \\
\hline $22 q$ & & & & & & & & & & & & & & & & & & & & & & & & $22 \mathrm{q}$ \\
\hline$X p$ & & & & & & & & & & & & & & & & & & & & & & & & $X p$ \\
\hline$X q$ & & & & & & & & & & & & & & & & & & & & & & & & $\mathrm{Xq}$ \\
\hline $\mathrm{Y}$ & $=$ & $=$ & 1 & $\bar{E}$ & 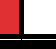 & \pm & $\amalg$ & 1 & $=$ & $\perp$ & 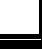 & \pm & 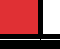 & $=$ & $=$ & 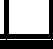 & ב & $=$ & $=$ & 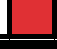 & $=$ & & $\perp$ & $\mathrm{Y}$ \\
\hline sex & $\mathrm{m}$ & $\mathrm{m}$ & \begin{tabular}{l|l}
$\mathrm{m}$ & $\mathrm{r}$ \\
\end{tabular} & $\mathrm{m} / \mathrm{m}$ & $\begin{array}{ll}n & f \\
\end{array}$ & $\mathrm{~m}$ & $\mathrm{~m}$ & \begin{tabular}{l|l}
$f$ & $m$ \\
\end{tabular} & $\mathrm{~m}$ & $\mathrm{~m}$ & $f$ & $\mathrm{~m}$ & \begin{tabular}{l|l}
$m$ & $n$ \\
\end{tabular} & $\mathrm{~m} / \mathrm{m}$ & n $f$ & $f$ & $\mathrm{~m}$ & \begin{tabular}{l|l}
$f$ & $f$
\end{tabular} & 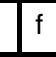 & $\mathrm{m}$ & $\mathrm{m}$ & \begin{tabular}{l|l}
$m$ & $f$ \\
\end{tabular} & m & \\
\hline
\end{tabular}

Figure 1 CGH: Chromosomal losses (red) and gains (green) in the short (p) and long (q) arms of chromosomes as detected by CGH. Monosomy 3 appears as strong risk factor for metastatic spread. Three patients without monosomy 3 developed subsequent metastases (193-97, 047-00, 226-97). 

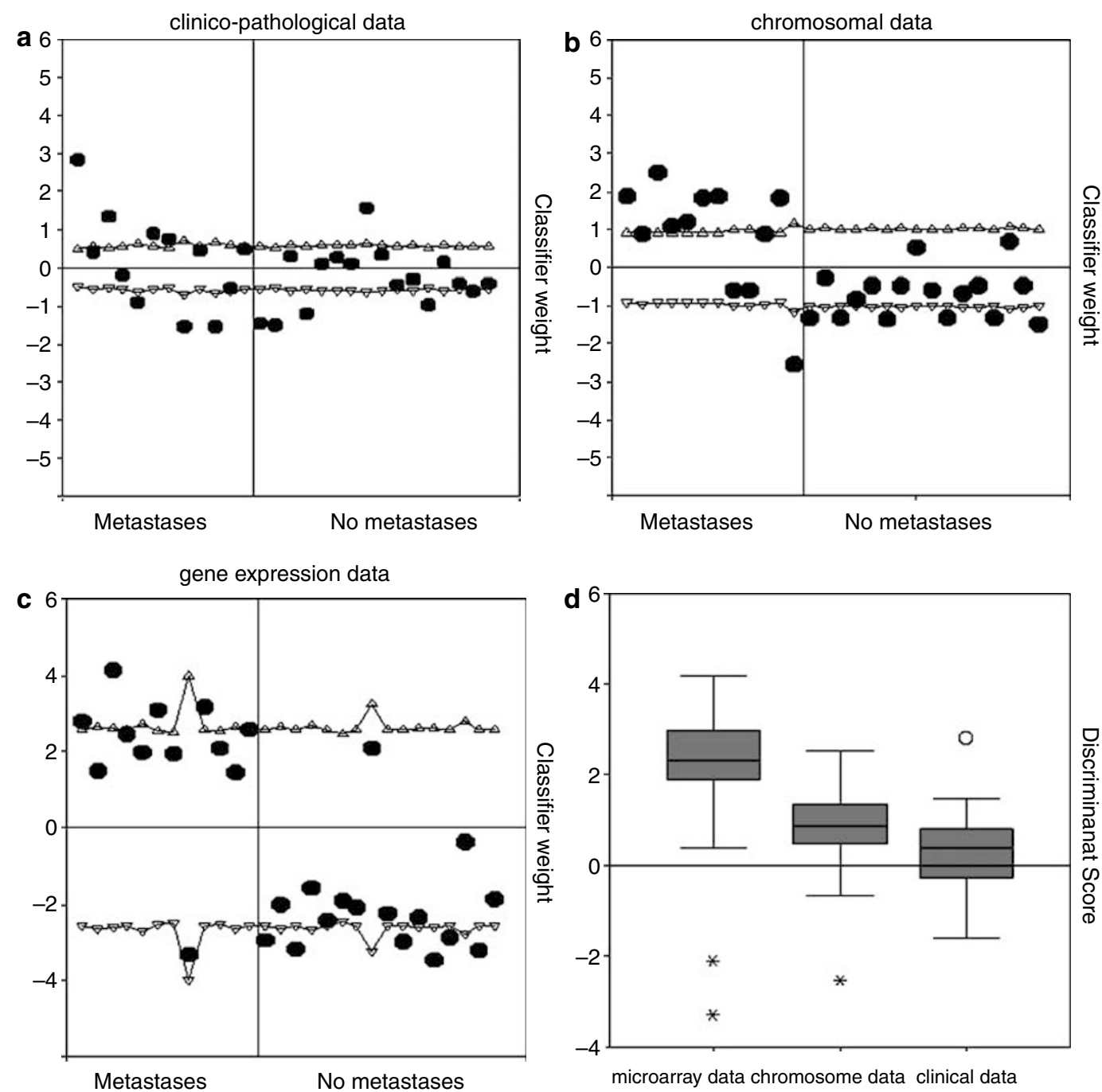

Figure 2 Dicriminant analysis (a-c): In panels A through C the upper and lower lines refer to the mean values of $M_{i j}(\boldsymbol{\nabla})$ and $N_{i j}(\boldsymbol{\Delta})$ as described in the Methods, the circles $(\mathbf{O})$ refer to the value of the classifier for the sample left out in the respective step. The zero line marks the cutoff between classification into metastases (upper half) and no metastases (lower half). In the displays correct/false classification and the distance to the cutoffs can be compared between different samples and different sets of prognostic variables. Samples were grouped into metastasizing and non-metastasizing uveal melanoma discriminated by negative and positive classifier value, respectively. From 28 samples 26 were classified according to subsequent developed metastases by gene expression data (c), 23 by chromosomal data (b) and 16 by clinico-pathological data (a). Grouping precision (d) Wilcoxon test for dependent samples was calculated between classifiers from gene expression, chromosomal and clinico-pathological data and yielded a significant ranking.

to the gene list associated with metastatic phenotype. ${ }^{14}$ Both gene lists were able to group our patients as high-risk or low-risk patients for subsequent metastases just as for our gene expression data. Both gene lists grouped 25 of 28 samples correctly (data not shown).

\section{Discussion}

The primary objective of our study was to investigate the precision of gene expression profiling in comparison to clinico-pathological and chromosomal data of uveal melanoma to assess the risk of development of subsequent metastases. Results of discriminant analyses were standardized to compare different sets of predictor variables, namely gene expression, $\mathrm{CGH}$ and clinico-pathological data.

In our series the primary uveal melanoma lesions were all large tumors, which are typically treated by enucleation or excision and thus yield adequate tissue for analysis. Assuming that enucleation and excision had no influence of the metastatic spread, both treatment groups were analyzed together. The analysis of the metastasesfree survival and the discriminant analyses based on clinico-pathological data demonstrated only a poor 


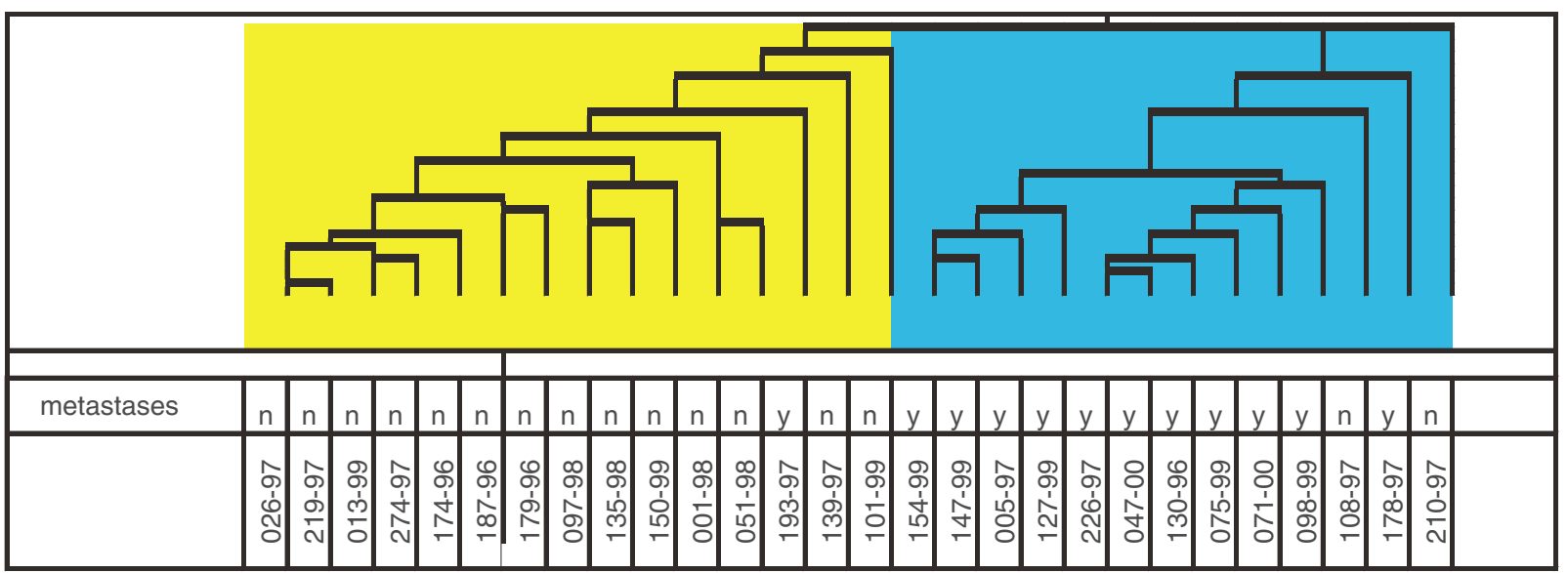

Figure 3 Grouping: Unsupervised analysis based on 8465 probe sets yielded a classification into two groups (groups a and b), with 12 of 11 patients with subsequent development of metastases (y) clustering in group A, and 14 of 15 patients without metastases (n) clustering in group b. Sample 210-97 was separately grouped.

prediction of metastatic spread. Gene expression data analyzed with diagonal linear discriminant analysis grouped patients according to their risk, misclassifying tumors from only two of 28 patients. Unsupervised learning (cluster analysis) revealed a correct grouping in 24 of 27 patients. Two samples (108-97 and 193-97) were misclassified in both analyses. Sample 210-97, which was a non-metastatic tumor with monosomy 3 was grouped into a separate third cluster, indicating a unique gene expression pattern. Interestingly, sample 210-97 was appropriately categorized as part of the cluster without subsequent metastases, when the 42 genes identified to discriminate high- from low-risk tumors for subsequent metastases were used to perform cluster analysis, suggesting that the pre-selected 42 classifying genes play a curial role in metastatic spread. CGH-based discriminant analysis of the same type grouped patients according to their risk for metastases with a significantly lower precision. Thus, in our hands gene expression profiling appeared to be the most suitable method for stratification of patients with high-risk uveal melanomas in prospective clinical trials, as has been suggested for a variety of hematopoietic ${ }^{17,18}$ as well as solid ${ }^{19-21}$ tumors.

Two other groups have investigated gene expression in uveal melanomas. The first report by Tschentscher $e a l^{13}$ defined the gene expression profile of tumors carrying monosomy 3 by presenting 201 significantly differentially expressed genes. Onken $e t a l^{14}$ described the prognostic value of gene expression analysis similar to our study identifying 62 significantly differentially expressed genes. Surprisingly, only one gene (phosphoinositide-3-kinase, regulatory subunit 4, p150) could be identified as differentially expressed in all three studies. However, all three gene lists were able to group our patient cohort better than all other risk factors for subsequent metastases.

Even if all three studies were of limited sample size, a significant grouping into only two clinical courses was possible in all studies reflecting the biological difference in metastasizing $v s$ non-metastasizing tumors. None of the studies found a third group by unsupervised hierarchical clustering indicating the homogeneity of the tumor biology. The consistently identified two-groupscluster allowed a statistically significant comparison even with a small sample size. Additionally, the population genetic differences between all three studies may contribute to the small overlap of differentially expressed genes. However, more critical appears to be the methodological variations of feature selection and different Affymetrix GeneChips. This exemplarily could be shown by using the same or different Affymetrix GeneChips in different laboratories to subgroup AML samples. ${ }^{18}$ Consistently samples were correctly grouped regardless of the used GeneChip or the laboratory. But importantly, lists of differentially expressed genes were substantially different indicating the issue of cutoff points for significantly differentially expressed genes. Defining classifying genes based on rank list appears problematic although associated with loss of information by setting a cutoff point to include and exclude probe sets. Furthermore, biological processes might be redundantly regulated by the expression of many different genes in different pathways. Accordingly, in our ocular melanoma study, the set of selected genes from our sample set worked, and the presented gene lists from Tschentscher and Onken also worked well on our sample 


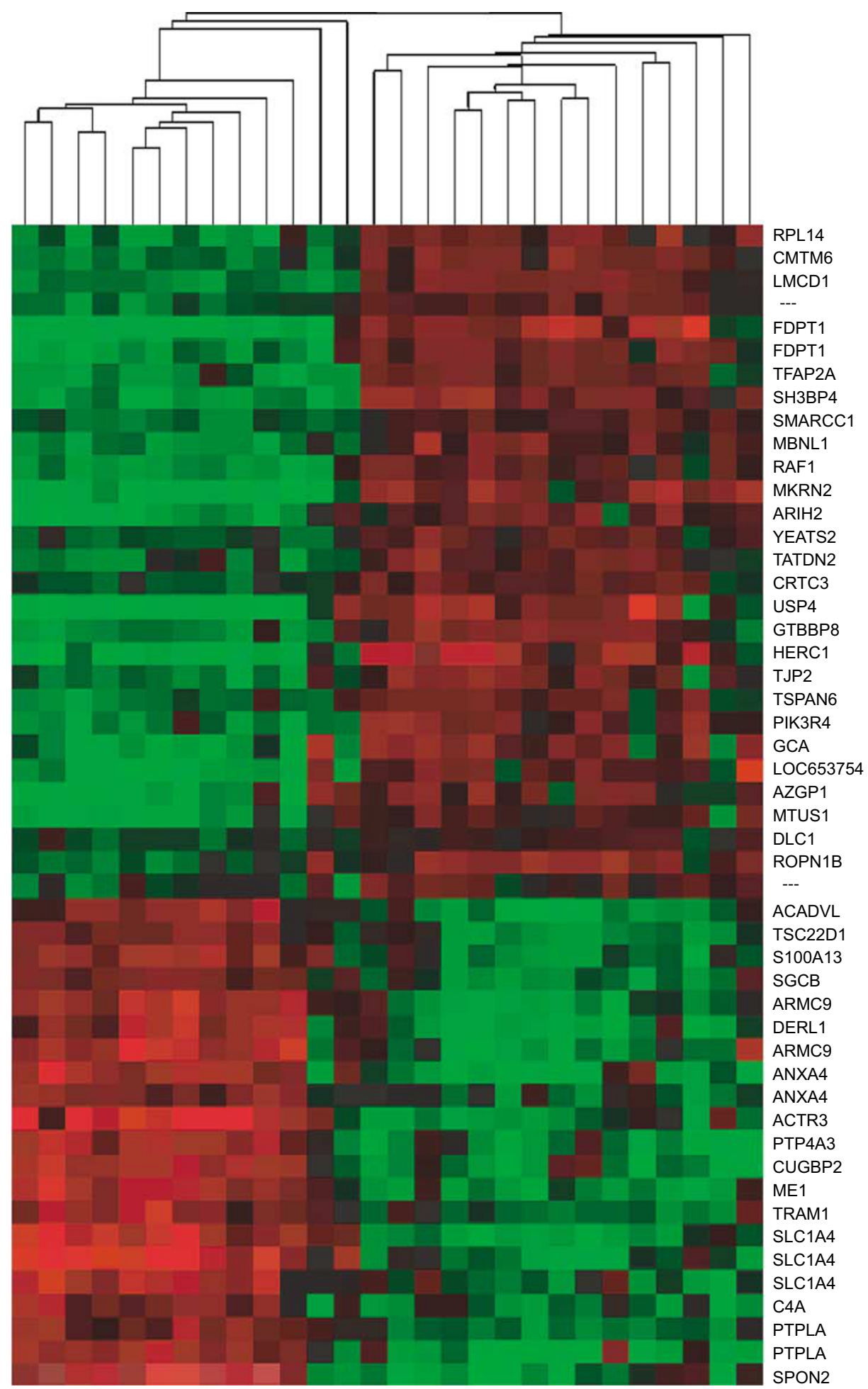

人ิ

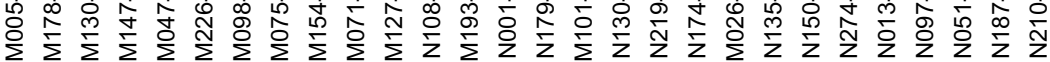

Figure 4 Gene expression profiling: Hierarchical cluster analysis based on 50 classifying probe sets determined by discriminant analysis (up (red) and down (green) regulated genes in metastasizing primary uveal melanoma). The choice of the 50 probe sets was not crossvalidated. 
set. However, because of the redundancy of regulation and different cutoff points to include and exclude genes from further analysis, list of selected genes within the sample sets do not overlap considerably.

All three studies showed, that the presence of monosomy 3 significantly influenced the expression profile of uveal melanoma. However, the complete data for all our patients demonstrated the ability of gene expression data to predict a high risk of metastases even in patients without the strong risk factor monosomy 3.

Taken together, gene expression profiling of our patient cohort provides a highly predictive pattern for the risk of metastases. Our observation suggests, that gene expression profiling can be used for stratification of patients in prospective clinical trials. Despite the considerable variability observed in three studies, however, different discriminating gene lists group patients more precisely according to their risk for subsequent metastases than chromosomal or clinicopathological data. Further, the identification of two genes linked to chromosomal alterations that are consistently predictive for metastases over 3 different analyses is of interest for detailed elucidation of the molecular basis of the metastatic process and for designing targeted treatment strategies.

\section{Acknowledgements}

The work was supported in part by the EORTC Melanoma Group.

\section{References}

1 Diener-West M, Reynolds SM, Agugliaro DJ, Caldwell R, Cumming K, Earle JD et al. Development of metastatic disease after enrollment in the COMS trials for treatment of choroidal melanoma: Collaborative Ocular Melanoma Study Group Report No. 26. Arch Ophthalmol 2005; 123: 1639-1643.

2 Assessment of metastatic disease status at death in 435 patients with large choroidal melanoma in the Collaborative Ocular Melanoma Study (COMS). COMS report No. 15. Arch Ophthalmol 2001; 119: 670-676.

3 Gragoudas ES, Egan KM, Seddon JM, Glynn RJ, Walsh SM, Finn SM et al. Survival of patients with metastases from uveal melanoma. Ophthalmology 1991; 98: 383-389; discussion 90.

4 Rietschel P, Panageas KS, Hanlon C, Patel A, Abramson DH, Chapman PB. Variates of survival in metastatic uveal melanoma. J Clin Oncol 2005; 23: 8076-8080.

5 Seddon JM, Albert DM, Lavin PT, Robinson N. A prognostic factor study of disease-free interval and survival following enucleation for uveal melanoma. Arch Ophthalmol 1983; 101: 1894-1899.

6 Mooy CM, De Jong PT. Prognostic parameters in uveal melanoma: a review. Surv Ophthalmol 1996; 41: 215-228.
7 Shammas HF, Blodi FC. Prognostic factors in choroidal and ciliary body melanomas. Arch Ophthalmol 1977; 95: 63-69.

8 Singh AD, Shields CL, Shields JA. Prognostic factors in uveal melanoma. Melanoma Res 2001; 11: 255-263.

9 Schmittel A, Bechrakis NE, Martus P, Mutlu D, Scheibenbogen C, Bornfeld N et al. Independent prognostic factors for distant metastases and survival in patients with primary uveal melanoma. Eur J Cancer 2004; 40: 2389-2395.

10 Keilholz U, Goldin-Lang P, Bechrakis NE, Max N, Letsch A, Schmittel A et al. Quantitative detection of circulating tumor cells in cutaneous and ocular melanoma and quality assessment by real-time reverse transcriptase-polymerase chain reaction. Clin Cancer Res 2004; 10: 1605-1612.

11 Callejo SA, Antecka E, Blanco PL, Edelstein C, Burnier MN. Identification of circulating malignant cells and its correlation with prognostic factors and treatment in uveal melanoma. A prospective longitudinal study. 2006, published 31 March 2006 [E-pub ahead of print] doi:10.1038/sj.eye.6702322

12 Prescher G, Bornfeld N, Hirche H, Horsthemke B, Jockel $\mathrm{KH}$, Becher R. Prognostic implications of monosomy 3 in uveal melanoma. Lancet 1996; 347: 1222-1225.

13 Tschentscher F, Husing J, Holter T, Kruse E, Dresen IG, Jockel $\mathrm{KH}$ et al. Tumor classification based on gene expression profiling shows that uveal melanomas with and without monosomy 3 represent two distinct entities. Cancer Res 2003; 63: 2578-2584.

14 Onken MD, Worley LA, Ehlers JP, Harbour JW. Gene expression profiling in uveal melanoma reveals two molecular classes and predicts metastatic death. Cancer Res 2004; 64: 7205-7209.

15 Tonnies H, Poland J, Sinha P, Lage H. Association of genomic imbalances with drug resistance and thermoresistance in human gastric carcinoma cells. Int J Cancer 2003; 103: 752-758.

16 Dudoit S, Fridlyand J. Classification in s experiments. In: Speed, T (ed). Statistical Analysis of Gene Expression Microarray Data. 102 Chapman \& Hall/CRC: London, 2003.

17 Ross ME, Mahfouz R, Onciu M, Liu HC, Zhou X, Song G et al. Gene expression profiling of pediatric acute myelogenous leukemia. Blood 2004; 104: 3679-3687.

18 Kohlmann A, Schoch C, Schnittger S, Dugas M, Hiddemann $\mathrm{W}$, Kern W et al. Molecular characterization of acute leukemias by use of microarray technology. Genes Chromosomes Cancer 2003; 37: 396-405.

19 Freije WA, Castro-Vargas FE, Fang Z, Horvath S, Cloughesy $\mathrm{T}$, Liau LM et al. Gene expression profiling of gliomas strongly predicts survival. Cancer Res 2004; 64: 6503-6510.

20 Haven CJ, Howell VM, Eilers PH, Dunne R, Takahashi M, van Puijenbroek $\mathrm{M}$ et al. Gene expression of parathyroid tumors: molecular subclassification and identification of the potential malignant phenotype. Cancer Res 2004; 64: 7405-7411.

21 Ginos MA, Page GP, Michalowicz BS, Patel KJ, Volker SE, Pambuccian SE et al. Identification of a gene expression signature associated with recurrent disease in squamous cell carcinoma of the head and neck. Cancer Res 2004; 64: 55-63.

\section{Appendix}

Details of the primary supervised learning procedure (applied $28 * 3=84$ times)

1. Select sample $i(i=1, \ldots, 28)$ and a complete subset of prognostic variables $j(j=1$ all micro array data, $j=2$ 
all chromosomal data, $j=3$ all clinico-pathological data).

2. Construct from the 27 samples (sample $i$ excluded) a linear classifier $c_{i j}(i=1, \ldots, 28, j=1,2,3)$ using DLDA.

3. Construct a standardized classifier $C_{i j}$ from $c_{i j}$ according to the following criteria: The mean value for patients with metastases, $M_{i j}$, is positive, the mean value for patients without metastases, $N_{i j}$, fulfills $N_{i j}=-M_{i j}$, and the pooled variance within patient groups, $\sigma_{i j}^{2}$, is set equal to 1 .

4. Store $C_{i j}, M_{i j}$, and $N_{i j}$. For each $i$, apply the classifiers $C_{i j}(j=1,2,3)$ to patient $i$ only and store the value of $C_{i j}$ and the classification result.
An overall comparison between the gene expression, chromosomal and clinico-pathological classifiers was performed using $D_{i j}=C_{i j}$ as described above for patients with metastases and $D_{i j}=-C_{i j}$ for patients without metastases. Thus, positive values of $D_{i j}$ correspond to a correct classification for patients, both with and without metastases. The size of $D_{i j}$ further quantifies the confidence of this classification. The values $D_{i j}$ were compared between the three sets of prognostic variables using the Wilcoxon test for paired samples. A set $j$ of prognostic variables is superior to a set $j^{\prime}$ if the values $D_{i j}$ are significantly larger than the values $D_{i j}^{\prime}$. 